\title{
Evidence for up-to-date clinical dental practice. A review of 10 years of the Cochrane Oral Health Group
}

\author{
Derek Richards \\ Editor, Evidence-based Dentistry
}

The end of May this year saw the Editor participating in a conference at the

Manchester Conference Centre to celebrate the 10th anniversary of the Cochrane Oral Health Group's (COHG) presence at Manchester Dental School.

Evidence-Based Dentistry (2003) 7, 60-61. doi:10.1038/sj.ebd.6400421
The meeting was opened by Professor Bill Shaw, one of the Group's co-ordinating editors and my tutor at dental school. Professor Shaw outlined his early interest in trials in cleft lip and palate and how his future research directions were influenced by meeting, Sir Iain Chalmers (the first Director of the Cochrane Collaboration) and Archie Cochrane. Professor Shaw also recalled the original registration of the COHG in the US under the co-ordinating editorship of Alexia Antczak Bouckoms. When in 1996 Alexia was unable to continue to lead the Group, the editorial base transferred to Manchester, to the university's Department of Dental Medicine and Surgery. Here, Bill and Professor Helen Worthington became co-ordinating editors.

At the meeting, Professor Worthington followed, with a presentation describing the achievements of the first 10 years: the COHG now has 54 reviews and 68 protocols in the third issue of the Cochrane Library this year. Eighteen more protocols are currently undergoing peer review and a further 35 titles are registered (for further details see the group's website, www.ohg.cochrane.org/).

Stephen Hancocks gave a very entertaining talk, the core of which has since been published as an editorial in the British Dental Journal, ${ }^{1}$ both of which raise

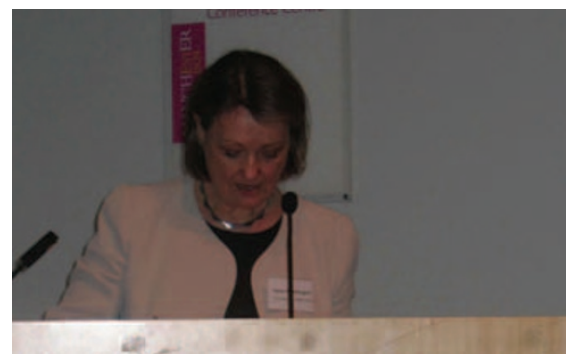

Figure 1. Professor Worthington addressing the conference important issues about the dental research agenda and what researchers do. After Stephen spoke, Jim Bader, Research Professor in the Operative Department at the University of North Carolina in the US, then gave an international perspective of the activities of the COHG: Using his survey of systematic reviews as a starting point, he highlighted the limited number of definitive answers that have resulted from systematic reviews so far. He highlighted the slow conversion rate of protocols and the need to maintain the flow of new reviews while improving the utility of the review to practitioners. Using Google and other mainstream sites, he emphasised how Cochrane reviews of dental topics have limited visibility there, to both public and professionals, and the need therefore to improve both visibility and utilisation. The thorny issue of implementation of the evidencebased approach was also raised, along with the challenges that implementing change might bring. Professor Bader concluded by suggesting a number of approaches that might be effective, for example evidencebased assessment for re-licensure or revalidation, the measurement of evidence-based performance by process or outcome measure, or reimbursement/ payment for appropriate use of the evidence-based approach.

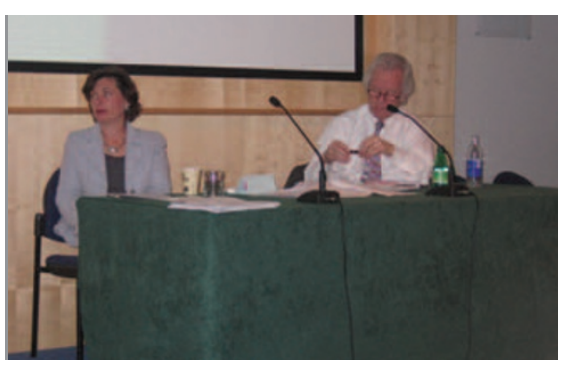

Figure 2. Sir Muir Gray and Dr Jan Clarkson at the podium
Sir Iain Chalmers closed the opening session. Not only the former director of the Cochrane Collaboration, he is the current Editor of the James Lind Library (www. jameslindlibrary.org). The latter was created to help people understand fair tests of treatments in healthcare, so Sir Iain's talk illustrated how fair tests have developed over the centuries. This was a very interesting and balanced overview of his involvement in the systematic review of water fluoridation. He restated clearly the findings of the review and did not hide his disappointment at how the predominant view within the dental profession has been that the results clearly vindicated its stance. Sir Iain's own view is that is to have definitive evidence of the effects of water fluoridation more high quality research still needs to be carried out - I firmly agree. Following the results of the systematic review $^{3}$ the dental profession should have been calling for this research to be funded rather than repeating the old rhetoric

The next sessions of the conference involved a series of presentations that outlined the work carried out within the various specialities over the past 10 years, what evidence is currently available and what is in progress. The presentations were all given by individuals who have participated in Cochrane reviews and provided a more

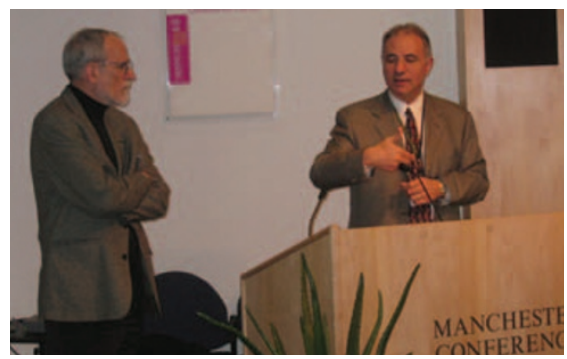

Figure 3. Professors Bader and Ismail taking feedback from the small groups 


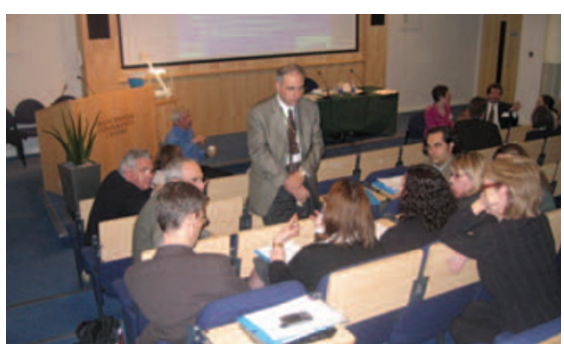

Figure 4. Workshop groups in action

detailed overview of what has been achieved by the COHG since its establishment. The areas covered were as follows:

- Caries prevention (Valeria Marinho, Queen Mary's School of Medicine and Dentistry, London, UK)

- Restorative/ endodontics (Paul Brunton, School of Dentistry, University of Manchester, UK)

- Periodontology (Richard Tucker, University College London-Eastman Dental Institute, London, UK)

- Implantology and Prosthetics(Marco Esposito, School of Dentistry, University of Manchester, UK)

- Orthodontics (Jayne Harrison, Liverpool University Dental Hospital, UK)

- Oral medicine Joanna Zakrzewska, Queen Mary's School of Medicine and Dentistry, London, UK)

- Dental public health (Peter Robinson, School of Clinical Dentistry, University of Sheffield, UK)

- Oral surgery/ pain relief (Paul Coulthard, School of Dentistry, University of Manchester, UK)
The final speaker was Professor Amid Ismail (School of Dentistry, University of Michigan, Ann Arbor, Michigan, USA) who described the American Dental Association's (ADA) developing role in the promotion of evidence-based dentistry. The ADA increasingly emphasises evidence-based dentistry, a large measure of which may be credited to the advocacy of Professor Ismail. He outlined some of their future plans, which can be seen on the ADA website (www.ada.org/ prof/resources/topics/evidencebased.asp). typesetter: break where necessary The very enjoyable and informative day was brought to a close by Professor Shaw.

The second day was largely broken into workshop sessions that examined the gaps in evidence and the barriers to dissemination and implementation found in various clinical areas highlighted on the first day. Between the two workshop sessions we were treated to two enjoyable presentations, the first by Sir Muir Gray, the Director of the National Library for Health. Muir used the analogy of the need for pure clear water with the need for pure clean knowledge and noted that, "Professionals and patients need clean clear knowledge for decision making just as they need clean clear water for hand washing", and that knowledge like water, "needs to be filtered, and sometimes purified". He also highlighted the need for organisations to have a board-level Knowledge Officer ${ }^{4}$ because of the power of knowledge. He challenged the audience to develop a clear vision of where dentistry should be in the year 2010. This challenge was accepted by the audience and formed part of one of the feedback sessions.
The second presentation was an entertaining and informative look by Jan Clarkson at the implementation of research evidence. This took us from the reason she became involved with the COHG to her latest research on implementing evidence-based practice in primary dental care, the ERUPT trial, ${ }^{5}$ highlighting opportunities and challenges for the future.

The feedback from the afternoon workshops on barriers to dissemination and implementationwas ably chaired by Professors Bader and Ismail. This included the section on the challenge from Muir Gray on where we should be by the year 2010, with Professor Nigel Pitts giving an impromptu perspective on how this might all fit together. The formal proceedings were closed by Professor Shaw before many of the participants attended methodology sessions that dealt with critical appraisal, information searching and systematic reviews and the COHG.

1. Hancocks S. Asking the right questions. Br Dent J 2006; 200:649.

2. Bader J, Ismail A. Survey of systematic reviews in dentistry. J Am Dent Assoc 2004; 135:464-473.

3. McDonagh MS, Whiting PF, Wilson PM, et al. Systematic review of water fluoridation. Br Med J 2000; 321:855-859.

4. Gray JA. Where's the chief knowledge officer? To manage the most precious resource of all. Br Med ] 1998; 317:832.

5. Clarkson J, Turner S, Bonetti D, et al. Implementation $\mathrm{RCT}$ of caries prevention treatment and caries risk. International Association for Dental Research 84th General Session, Brisbane,June 2006. 